\title{
High Molecular Weight Polymer Promotes Bone Health and Prevents Bone Loss Under Salmonella Challenge in Broiler Chickens
}

\begin{abstract}
Sandi Raehtz', Billy M. Hargis ${ }^{2}$, Vivek A. Kuttappan ${ }^{2}$, Rifat Pamukcu ${ }^{3}$, Lisa R. Bielke ${ }^{4 \dagger}$ and Laura R. McCabe ${ }^{1,5,6 * t}$

' Department of Physiology, Michigan State University, East Lansing, MI, United States, ${ }^{2}$ Department of Poultry Science, University of Arkansas, Fayetteville, AR, United States, ${ }^{3}$ Midway Pharmaceuticals, Spring House, PA, United States, ${ }^{4}$ Department of Animal Science, Ohio State University, Columbus, OH, United States, ${ }^{5}$ Department of Radiology, Michigan State University, East Lansing, MI, United States, ${ }^{6}$ Biomedical Imaging Research Centre, Michigan State University, East Lansing, MI, United States
\end{abstract}

\section{OPEN ACCESS}

Edited by:

Rocco Victor Carsia,

Rowan University School of Osteopathic Medicine, United States

Reviewed by:

Toshie Sugiyama,

Niigata University, Japan Michael Kogut

Agricultural Research Service (USDA), United States

*Correspondence: Laura R. McCabe mccabel@msu.edu

†These authors have contributed equally to this work and co-senior authors.

Specialty section:

This article was submitted to Avian Physiology,

a section of the journal

Frontiers in Physiology

Received: 11 September 2017

Accepted: 28 March 2018

Published: 13 April 2018

Citation:

Raehtz S, Hargis BM, Kuttappan VA

Pamukcu R, Bielke LR and

McCabe LR (2018) High Molecular Weight Polymer Promotes Bone

Health and Prevents Bone Loss Under Salmonella Challenge in Broiler Chickens. Front. Physiol. 9:384. doi: 10.3389/fphys.2018.00384
As a consequence of rapid growth, broiler chickens are more susceptible to infection as well as bone fractures that result in birds being culled. Intestinal infection/inflammation has been demonstrated to promote bone loss in mice and humans. Given this link, we hypothesize that therapeutics that target the gut can benefit bone health. To test this, we infected broiler chickens (7 days old) with Salmonella and treated the birds with or without MDY, a non-absorbable mucus supplement known to benefit intestinal health, from day 1-21 or from day 14-21. Chicken femoral trabecular and cortical bone parameters were analyzed by microcomputed tomography at 21 days. Birds infected with Salmonella displayed significant trabecular bone loss and bone microarchitecture abnormalities that were specific to the femoral neck region, a common site of fracture in chickens. Histological analyses of the chicken bone indicated an increase in osteoclast surface/bone surface in this area indicating that infection-induced bone resorption likely causes the bone loss. Of great interest, treatment with MDY effectively prevented broiler chicken bone loss and architectural changes when given chronically throughout the experiment or for only a week after infection. The latter suggests that MDY may not only prevent bone loss but reverse bone loss. MDY also increased cortical bone mineral density in Salmonella-treated chickens. Taken together, our studies demonstrate that Salmonella-induced bone loss in broiler chickens is prevented by oral MDY.

Keywords: bone, osteoporosis, poultry, microbiome, intestine, gut, Salmonella, femoral-neck

\section{INTRODUCTION}

In the US, in 2010, approximately 36.9 billion pounds of broilers were sold for a retail value of 45 billion dollars and 6.8 billion pounds were exported (according to uspoultry.org). Over the years, chickens have been bred to grow rapidly to quickly obtain large muscle mass to increase market weight. This rapid growth has contributed to bone abnormalities, deformities and fractures (Vestergaard and Sanotra, 1999; Butterworth et al., 2001; Kestin et al., 2001; Knowles et al., 2008; Olkowski et al., 2011). The most common site of bone pathology is at the femur, specifically the 
femoral neck, which results in bird lameness (McNamee et al., 1999; McNamee and Smyth, 2000; Dinev, 2009; Durairaj et al., 2009; Olkowski et al., 2011). Selection for high growth rates is also linked to the development of chickens with decreased immune function which puts the birds at increased risk for bacterial infections (McNamee et al., 1999; Corr et al., 2003). Bacterial infections and bone loss/degeneration are major health concerns in broiler chickens that lead to economic losses of hundreds of millions of dollars annually (Cook, 2000).

Intestinal infection and it's associated inflammation are known to promote bone loss in mouse models and in patients with inflammatory bowel disease (Lin et al., 1996; Sylvester, 2005; Hamdani et al., 2008; Harris et al., 2009; Irwin et al., 2013, 2016; Sylvester et al., 2013). Studies in mice indicate that the extent of intestinal inflammation directly correlates with bone density loss (Irwin et al., 2016) and is linked to suppressed bone formation (Harris et al., 2009; Irwin et al., 2013). Correspondingly, removing the mediator of intestinal inflammation results in bone density recovery (Harris et al., 2009). In avian models, the link between enteric inflammation and bone health has not been directly examined, but several reports indicate an association (Huff et al., 2006; Tellez et al., 2014; Wideman, 2016; Bielke et al., 2017). For example, broiler chickens fed a rye diet display mucosal damage, increased intestinal viscosity and permeability, and lowered bone strength and mineralization (Tellez et al., 2014). Rapidly growing turkeys exhibit altered stress responses and increased chronic bacterial diseases such as turkey osteomyelitis complex (Huff et al., 2006) similar to rapidly growing broiler chickens which display altered immune function, bacterial infection and bone deformation (McNamee et al., 1999; Corr et al., 2003). Pro-inflammatory response are associated with bone loss in many species including broiler chickens (Mireles et al., 2005). Thus, broiler chickens are at increased risk for infection and possibly decreased bone health as a consequence of pressures for increased growth, largeproduction facilities, and decreased antibiotic use (Cook, 2000; Mehaisen et al., 2017).

Salmonella infection in chickens can cause mild enteric inflammation characterized by increased mRNA expression of proinflammatory cytokines including interleukin-6 (IL-6), IL-8, IL-12, LPS-induced tumor necrosis alpha factor (LITAF), and interferon gamma (IFN- $\gamma$ ) (Kaiser et al., 2000; Withanage et al., 2005; Haghighi et al., 2008; Quinteiro-Filho et al., 2010, 2012; Higgins et al., 2011; Setta et al., 2012; Kubota et al., 2013). Salmonella infection in mammals also causes inflammation (Eckmann and Kagnoff, 2001), however the specific cytokine responses are quite different between rodents and chickens. Ingestion of selected probiotics, which can promote immune quiescence in the intestine, can reduce intestinal inflammation (Haghighi et al., 2008; Neish, 2009). In fact, a commercially used poultry probiotic selected for anti-Salmonella activity has been shown to induce changes in expression of genes related to lipopolysaccharide (LPS) response, NF- $\kappa$, and apoptosis pathways in vivo and in vitro (Higgins et al., 2011; Carey and Kostrzynska, 2013) in response to Salmonella typhimurium challenge. The exact factors that cause this effect are not yet known.
MDY (MDY-1001, Midway Pharmaceuticals) is a nonabsorbed, non-metabolized derivative of a high molecular weight polyethylene glycol (PEG) polymer averaging $15 \mathrm{kDa}$ in size. PEG derivatives are hydrophilic molecules which have been used in medicine for several years. Short chain length PEG molecules have oncotic properties that are favorable for absorbing water molecules and reducing tissue edema as well as acting as a reservoir for radical oxygen species (ROS) preventing cell membrane damage (Koob and Borgens, 2006; Luo and Shi, 2007). Because of these properties, PEG derivatives have been used in bowel prep solutions (GoLytely; Braintreelabs) and pharmaceutical drug delivery (pegylated compounds). Additionally, certain PEG molecules can increase epithelial cell tight junctions and mucus barrier function resulting in a preserved epithelial cell barrier (Moeser et al., 2008; Teramura et al., 2008; Valuckaite et al., 2009; Oltean et al., 2012, 2015). High molecular weight PEGs, like MDY, can associate with the lipid rafts of cell membranes (Valuckaite et al., 2009). They can also prevent pathogenic bacteria (Pseudomonas aeruginosa) adherence to the epithelial barrier and prevent subsequent sepsis and death in mice (Wu et al., 2004). High molecular weight PEG has also been shown to promote organ transplant preservation and prevent radiation induced mucosal damage (Valuckaite et al., 2009, 2013; Oltean et al., 2012, 2015).

Salmonella is known to infect mouse and human osteoblasts, cause bone and joint infection (osteomyelitis) clinically (Alexander et al., 2001; Marriott, 2013), promote inflammatory bone loss (Marriott, 2013), and promote enteric inflammation (Kaiser et al., 2000; Eckmann and Kagnoff, 2001; Withanage et al., 2005; Haghighi et al., 2008; Higgins et al., 2011; Quinteiro-Filho et al., 2012; Kubota et al., 2013). While the above studies report effects of paratyphoid Salmonella, several studies have reported effects of a specific Salmonella serovars, Salmonella Enteritidis, in chickens (Kogut et al., 1994, 1995; Stabler et al., 1994; Kaiser et al., 2000). Based on the link between gut and bone health (Mccabe et al., 2013; McCabe et al., 2015; Irwin et al., 2016; Collins et al., 2017a,b; Rios-Arce et al., 2017; Schepper et al., 2017), we hypothesized that Salmonella infection could have negative effects on broiler chicken skeletal health. Furthermore, given the effect of high molecular weight PEGs in strengthening the intestinal barrier (Teramura et al., 2008; Valuckaite et al., 2009, 2013; Oltean et al., 2012, 2015), we hypothesized that MDY may benefit the bone health of broiler chicks infected with Salmonella. Our studies support this premise.

\section{METHODS}

\section{Experimental Design}

Broiler chickens at 1-day-old were equivalently weightdistributed into the following treatment groups: (1) control $(n=24)$, (2) control + MDY for 3-weeks $(n=24)$, (3) Salmonella-infected $(n=24)$, (4) Salmonella-infected + MDY for 3-weeks $(n=24)$, and (5) Salmonella-infected + MDY for 1 -week $(n=15)$. For all Salmonella groups, birds at day 7 were infected by gavage with Salmonella Enteritidis (a total of $10^{6}$ colony forming units (cfu) in $0.25 \mathrm{ml}$ ). For MDY treatment groups, birds were given ad libitum feed containing 0.33\% MDY 
starting either on day 1 (3-week treatment; days 1 through 21; groups 2 and 4) or on day 14 (1-week treatment; only days 14-21; group 5) (Figure 1A). MDY (MDY-1001) is a high molecular weight PEG derivative being developed and generously provided by Midway Pharmaceuticals. Specifically, MDY is non-immunogenic and composed of 2 PEG chains crosslinked by a phenolic linker. The average MW of the MDY is $15 \mathrm{kDa}$. On day 21 of the study, the birds were humanely killed by carbon dioxide inhalation. Legs were removed at the hip joint and the femora isolated and fixed in formalin for 1 week and then transferred to $70 \%$ ethanol. All animal handling procedures were in compliance with the Institutional Animal Care and Use Committee at the University of Arkansas.

\section{Salmonella Culture}

A primary poultry isolate of Salmonella Enteritidis (SE), phage type $13 \mathrm{~A}$, was used to infect the chickens. This strain is associated

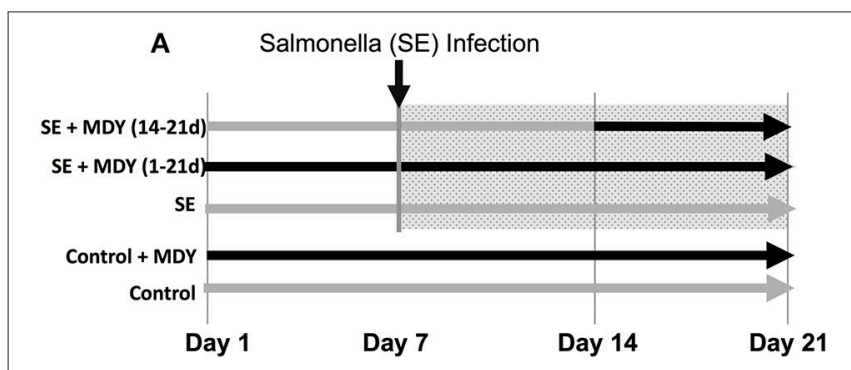

B

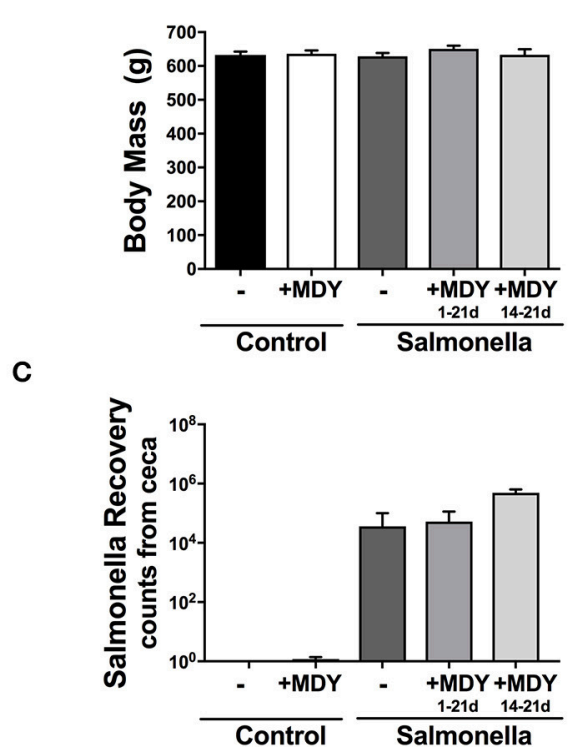

FIGURE 1 | Experimental design and chicken body mass. (A) 1-day-old chicks were weighed and evenly distributed into 5 groups: Control, control + $0.33 \% \mathrm{MDY}$ in feed (1-21d), Salmonella-infected, Salmonella-infected + $0.33 \%$ MDY in feed (1-21d), and Salmonella-infected + 0.33\% MDY in feed (14-21d). The birds were harvested on day 21. (B) Chick body mass was measured on experimental day 21. (C) Salmonella recovery rates from chicken ceca. Values represent averages \pm SE. $N \geq 8$. with decreased performance and increased enteric inflammation (Higgins et al., 2011; Quinteiro-Filho et al., 2012). The bacterium was originally obtained from the National Veterinary Services Laboratory (Ames, Iowa). For the study, Salmonella was grown in tryptic soy broth for approximately 8 hours. The cells were washed three times with $0.9 \%$ sterile saline by centrifugation $(1,820 \times \mathrm{g})$, and the approximate concentration of the stock solution was determined spectrophotometrically. The stock solution was serially diluted and confirmed by colony counts of three replicate samples $(0.1 \mathrm{~mL} /$ replicate $)$ that were spread plated on tryptic soy agar plates.

\section{Microcomputed Tomography}

Femora were scanned using a GE Explore Locus microcomputed tomography system with a voxel resolution of $20 \mu \mathrm{m}$ obtained from 400 views. Beam strength was set at $80 \mathrm{kV}$ and $450 \mu \mathrm{A}$ with a beam angle increment of 0.5 . Each scan consisted of 2 femurs placed in a $50 \mathrm{~mL}$ tube filled with ethanol and were scanned with a phantom bone used for calibration between scans. A fixed threshold of 376 was used to separate bone from bone marrow. Regions of interest (ROI) were identified and analyzed for bone density parameters and isosurface images were constructed using GE Healthcare Microview software. Three areas of the femur were focused on: proximal femoral metaphysis, femoral diaphysis, and femoral neck as noted in Figure 2. The length of the bone was calculated and trabecular bone was measured for $10 \%$ of the total length of the bone starting at the growth plate and moving proximally. Femurs were reoriented so that the femoral neck was along the $y$ axis and a cylindrical tube ( $2 \mathrm{~mm}$ diameter $\times 4 \mathrm{~mm}$ length) was used to analyze trabecular bone starting at the growth plate and going $4 \mathrm{~mm}$ down toward the proximal end of the bone. Finally, cortical bone shape/density was measured by placing a $7 \times 7 \times 7 \mathrm{~mm}$ cube at the diaphysis at $50 \%$ of the total bone length. In addition, a $0.75 \mathrm{~mm}$ cube was used to measure only cortical bone in this region.

\section{Histology}

Femurs, fixed in formalin and subsequently transferred to $70 \%$ ethanol, were decalcified by soaking in 14\% EDTA for 9 days followed by a $2 \mathrm{~h}$ rinse with running tap water. Femurs were then processed on an automated Electron Excelsior tissue processor (Thermo Fischer Scientific) for dehydration, clearing and infiltration using an extended schedule secondary to size. Samples were embedded by hand on a flat surface to demonstrate a longitudinal section through the femoral neck and proximal femur. Midway down, the bone shaft was sectioned transversely for cross section of bone as well as bone marrow analysis. Paraffin blocks were sectioned at $4 \mu \mathrm{m}$ on a Reichert Jung 2030 rotary microtome (Leica, Wetzlar, Germany).

Osteoclast, osteoblast and adipocyte quantification was performed by staining for tartrate-resistant acid phosphatase (TRAP) activity with a hematoxylin counterstain per manufacturer protocol (387A-IKT; Sigma). At least 10 images at $40 \mathrm{X}$ magnification were taken at the femoral neck, excluding the growth plate area, for osteoclast and osteoblast quantification 


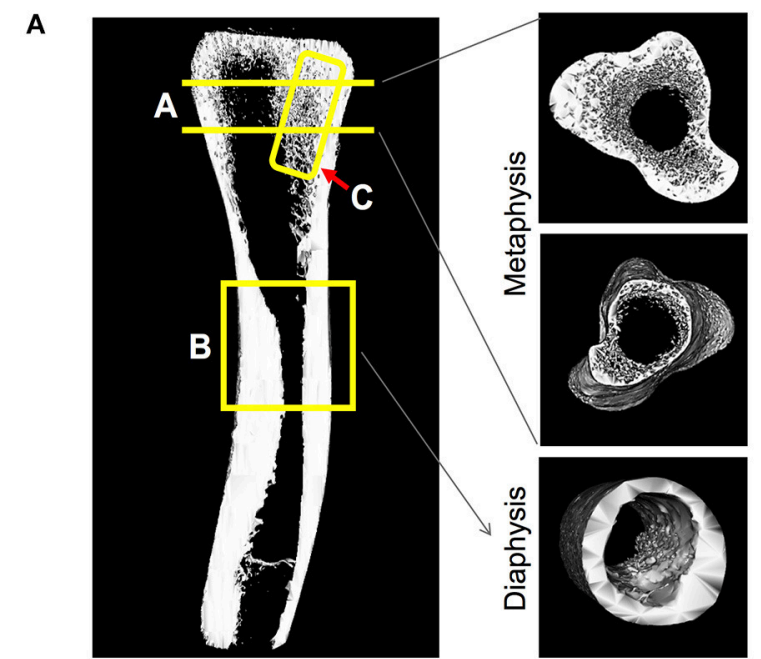

B

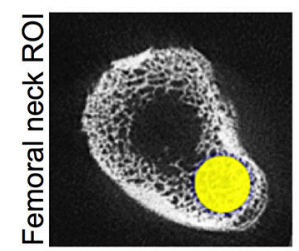

Proximal

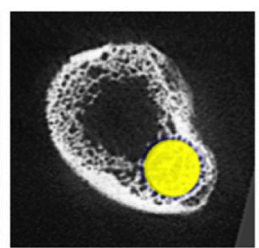

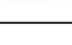

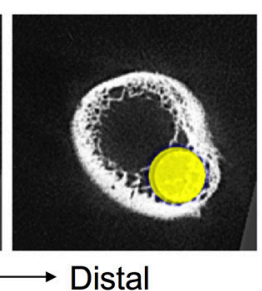

FIGURE 2 | Regions of chicken femoral bone analyzed by microcomputed tomography. (A) Isosurface image indicating regions of bone examined: A-Region $=$ trabecular bone in the metaphysis that is measured at $10 \%$ of the total femur length starting from the growth plate and moving distally. B-Region $=$ diaphyseal cortical bone that is measured at $50 \%$ of the total length of the femur by a $7 \mathrm{~mm}$ cube region of interest. C-Region $=$ femur neck trabecular bone region of interest (ROI) measured by utilizing a $2 \mathrm{~mm}$ diameter $\times 4 \mathrm{~mm}$ length cylindrical tube. (B) Three sections, proximal-to-distal, indicating the location of the cylinder used for the femur neck ROI.

and at least 5 images at $20 \mathrm{X}$ magnification were taken of the bone marrow for adipocyte measurements. Total bone surface measured averaged $1 \mathrm{~mm}$ or greater per image and did not differ between groups (data not shown). TRAP-positive osteoclast surface along the bone surface as well as osteoclast number was measured and expressed relative to the total bone surface measured. Osteoblasts counts were performed by cellular morphology and consisted of cuboidal cells in direct contact with the bone surface. The ratio of osteoclast number to osteoblast number (OC/OB ratio) was determined. The number of adipocytes in the bone marrow were measured using ImageJ (National Institute of Health, Bethesda, MD). Quantitation was performed blinded.

\section{Statistical Analysis}

All measurements are presented as the mean \pm SEM. Power analyses indicate that an $\mathrm{n}$ of 14 is sufficient to detect a $5 \%$ difference between groups (with an $\alpha$ of 0.05 and a power of $80 \%$ ) in bone measures. Significant outliers were removed using the Grubb's test for outliers. One-way ANOVA, with a Fischer post-hoc test as well as Pearson Correlations were performed using GraphPad Prism software version 6 (GraphPad, San Diego, CA, USA). $p$-values of $<0.05$ were considered significant and values of $<0.01$ were considered highly significant.

\section{RESULTS}

Broiler chickens at 7 days of age were infected enterically with Salmonella and supplemented with MDY (in the bird feed) beginning at day 1 (continuous) or day 14 (acute) (Figure 1A) to determine if MDY could improve chicken bone health. We infected the birds at this early stage of rapid growth since it is a time when the birds can be more susceptible to infection (Kebede, 2010) and under rapid growth the birds may exhibit a greater bone response to modeling dysregulation. At the time of necropsy, treatments did not appear to adversely affect body condition as there were no significant differences in body mass (Figure 1B) or femur length (data not shown) among groups. As expected, Salmonella levels were elevated in the ceca of the infected chickens (Figure 1C). It should be noted that while Salmonella was not detected in 22 out of the 24 control birds, 2 out of 24 control birds were positive for the presence of Salmonella, but the levels were barely detectable and well below levels seen in infected birds.

To examine bone density, we first focused on trabecular bone which is the most metabolically active bone region, and therefore is highly responsive and likely to show changes. Focusing on the femoral neck (Figure 2), where fractures often occur in chickens, we observed clear differences in trabecular bone structure between conditions as seen in the representative isosurface images (longitudinal and cross-sectional) shown in Figure 3A. Quantitation of the femoral neck trabecular bone volume fraction (BVF) revealed a nearly 20\% decrease in Salmonella infected birds. More importantly, infected birds that were treated with MDY, either from days 1-21 or from days 14-21, were protected from the Salmonella-induced bone loss (Figure 3B). Interestingly, femoral neck BVF in control birds treated with MDY trended to be increased relative to untreated controls, suggesting that MDY may support bone health in control animals. To test if the response was region specific, we examined the femoral metaphysis region and found that Salmonella infection did not reduce bone volume (Figure 3C). This finding implicates the femoral neck region as being more metabolically active and responsive to Salmonella infection and MDY treatment.

Additional measures of the femoral neck trabecular bone support the bone volume data. Specifically, bone mineral density (BMD) is decreased in Salmonella-infected birds and BMD and bone mineral content (BMC) are significantly increased in MDYtreated Salmonella-infected birds (Figures 4A,B). The trabecular bone architecture is also altered by infection resulting in trends to decrease trabecular thickness and correspondingly increase spacing between bone trabeculae, while treatment with MDY prevents the negative changes (Figures 4C,D). No changes were observed in trabecular number, indicating that a reduction of 
A
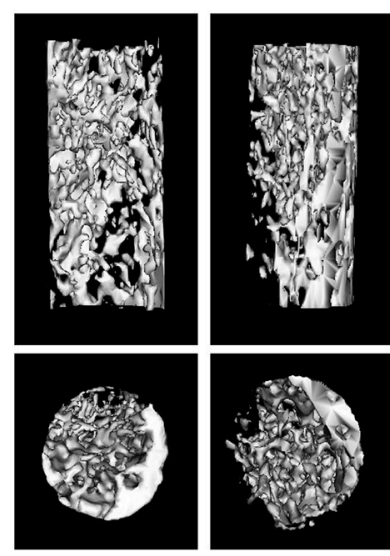

$-$

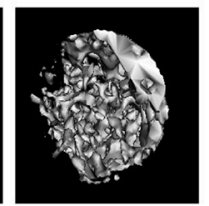

$+\mathrm{MDY}$

Control

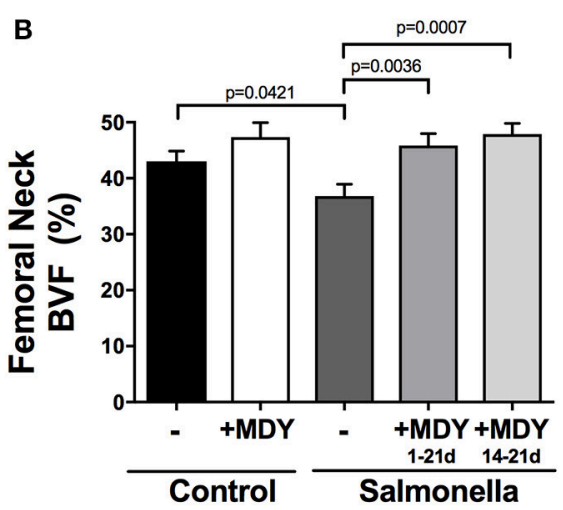

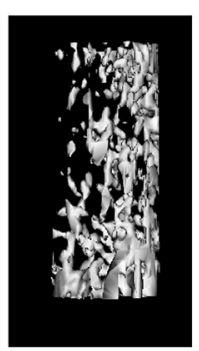
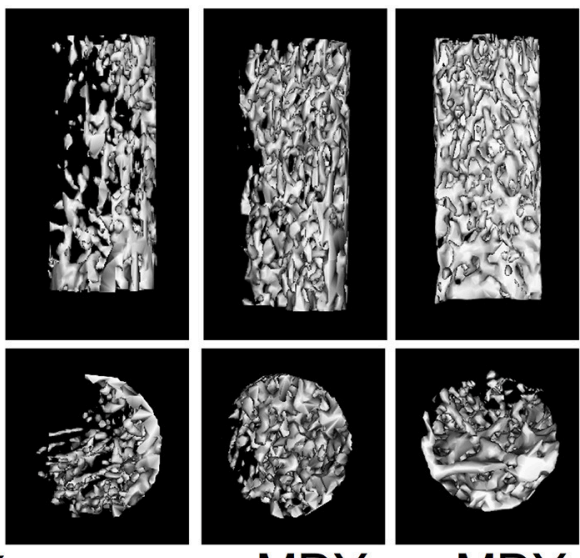

$+\mathrm{MDY}$

+ MDY

(1-21d)

(14-21d)

Salmonella infected

C

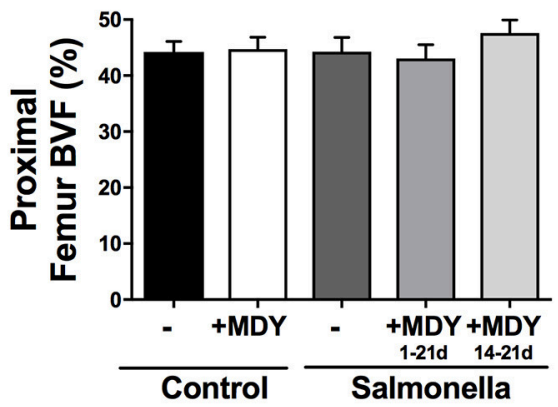

FIGURE 3 | Salmonella-induced bone loss specifically in the femoral neck region is prevented by MDY treatment. (A) Representative longitudinal and vertical isosurfaces of the chick femoral neck region. (B) Bone volume fraction (BVF) of the femoral neck. (C) Bone volume fraction of the proximal femur metaphysis. Values are means \pm SE. $N \geq 15$ per group. Lines represent statistical differences $(p<0.05)$ between bars at ends of line as determined by 1 -way ANOVA with a Fischer ad hoc test.

trabecular thickness is the predominant response to Salmonella infection in birds.

Next we examined the cortical bone of the femoral diaphysis. Though cortical BMD and BMC appear lower in Salmonellainfected birds than controls, the levels were not significantly different (Figure 5). This was true for other cortical bone parameters such as inner and outer perimeters, cortical area, marrow area, etc. (not shown), consistent with this region having less metabolic/modeling activity. However, MDY treatment, both the 1-21 and 14-21 day treatments, increased cortical BMD in Salmonella-infected birds (Figure 5).

As decreased bone volume can be a consequence of either increased resorption by osteoclasts or decreased formation by osteoblasts, we sought to examine the effect of Salmonella infection on these cell populations in the femoral neck using histomorphometry measures. Consistent with increased resorption, Salmonella-infected birds displayed an increase in TRAP-positive osteoclast surface with respect to both control and MDY-treated Salmonella-infected birds (Figures 6A,B). Osteoblast surface did not significantly change (data not shown). However, there was a significant increase in the ratio of osteoclast to osteoblast surface along the femoral neck trabecular bone in the Salmonella-infected birds relative to both control and MDY-treated Salmonella-infected birds (Figure 6C). Both the osteoclast surface as well as the ratio of osteoclasts to osteoblasts were significantly correlated to bone volume fraction (BVF\%) (Figures 6E,F). Quantiation of marrow adipocytes (derived from mesenchymal stem cells and thought to be inversely proportional to osteoblast numbers) revealed no significant difference between groups (Figure 6D), consistent with no observed change in osteoblast number.

\section{DISCUSSION}

While most poultry in the US have been grown under chronic sub-therapeutic antibiotic treatment, there has been a push 

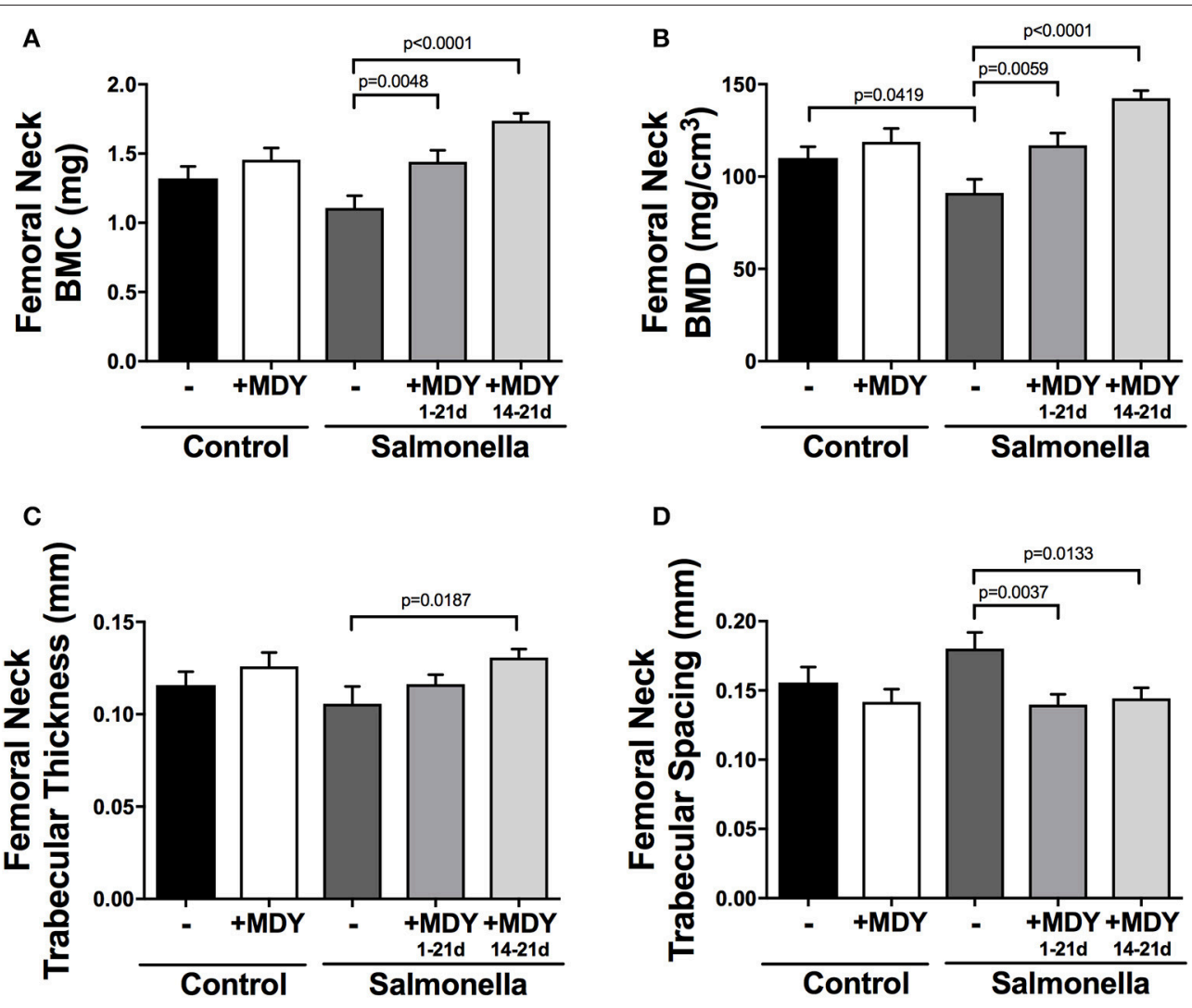

FIGURE 4 | Femoral neck trabecular bone parameters support MDY benefit to bone health in Salmonella-infected chicks. Shown are femoral neck trabecular bone parameters: (A) Bone mineral content (BMC), (B) Bone mineral density (BMD), (C) Trabecular thickness and (D) Trabecular spacing. Values are means \pm SE. $N \geq 15$ per group. Lines represent statistical differences $(p<0.05)$ between bars at ends of line as determined by 1 -way ANOVA with a Fischer ad hoc test.

for antibiotic-free chickens to reduce the risk of developing antibiotic resistant bacteria and to promote chemical-free chicken meat. With stressfull housing and the removal of chronic antibiotic treatment, chickens are more susceptible to infection (Cook, 2000). The inflammatory state may be an important contributor to decreased skeletal health (McNamee et al., 1999; Corr et al., 2003; Huff et al., 2006; Tellez et al., 2014; Wideman, 2016; Bielke et al., 2017) as suggested by mouse studies (Mccabe et al., 2013; McCabe et al., 2015; Irwin et al., 2016; Collins et al., 2017a,b; Rios-Arce et al., 2017; Schepper et al., 2017). The bone deformation or loss can result in fractures that cause the chickens to be culled, which costs the industry hundreds of millions of dollars annually (Cook, 2000). Here we show that Salmonella infection, on its own, is sufficient to cause broiler chicken femoral neck bone loss. We further demonstrate that MDY is an effective treatment to prevent broiler chicken bone loss.

Researchers indicate that up to $30 \%$ of broiler chickens suffer from gait defects (Knowles et al., 2008; Dinev, 2009). The femur is a key sight of bone degeneration that contributes to difficulty walking (McNamee and Smyth, 2000; Dinev, 2009; Durairaj et al., 2009; Olkowski et al., 2011). Olkowski et al. (2011) demonstrated that $85-90 \%$ of broiler chickens showing signs of lameness have pathology in the proximal end of the femur that typically involves changes in the femoral head including loss of subchondral bone, less organic matrix content, and in severe cases fractures. The results of these and similar studies led us to study Salmonella effects specifically on the femur. Our studies indicate that the femoral neck displays significant bone loss, changes in trabecular bone architecture, and an increase in the bone surface covered by osteoclasts as well as the ratio of osteoclasts to osteoblasts in Salmonella-infected birds. Interestingly, we saw no difference in bone density at the proximal femoral metaphysis or the femoral diaphysis. This location specificity is important to note, since benefits of other therapies on broiler chicken skeletal health may be missed if only proximal trabecular bone is examined. While we were not able to analyze other bones such as vertebrae, to determine if bone loss occurs at other skeletal sites, our findings are important because they demonstrate that infection and treatments can have varying responses within sites of a single bone.

Rodent disease models display bone loss in response to bacterial intestinal infection as well as inflammation secondary to colitis (Lin et al., 1996; Harris et al., 2009; Irwin et al., 2013). Our lab has also shown that low levels of inflammation from a surgical procedure (dorsal skin incision) is enough stimulus to cause bone loss in mice (Collins et al., 2016). Physiologic inflammation is typically controlled by the body to maintain homeostasis. However, activation of an immune response as seen 


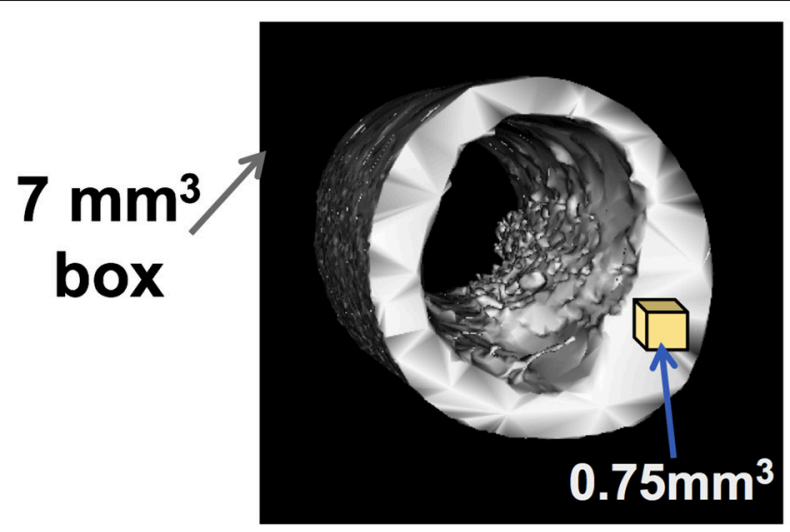

\begin{tabular}{|c|c|c|c|c|c|}
\hline \multirow[b]{2}{*}{ Cortical } & \multicolumn{2}{|c|}{ Control } & \multicolumn{3}{|c|}{ Salmonella } \\
\hline & - & + MDY & - & $\operatorname{MDY}(1-21 d)$ & $\operatorname{MDY}(14-21 d)$ \\
\hline \multicolumn{6}{|l|}{$\begin{array}{l}\text { Large cortical } \\
\text { volume }(7 \mathrm{~mm})\end{array}$} \\
\hline $\mathrm{BMD}\left(\mathrm{mg} / \mathrm{cm}^{3}\right)$ & $576 \pm 10$ & $557 \pm 14$ & $552 \pm 14$ & $589 \pm 11$ * & $646 \pm 5.2^{\wedge}$ \\
\hline BMC $(\mu \mathrm{g})$ & $39.4 \pm 1.9$ & $36.6 \pm 1.2$ & $37.7 \pm 1.8$ & $38.1 \pm 1.5$ & $44.5 \pm 2.0^{\wedge}$ \\
\hline \multicolumn{6}{|l|}{ Small cortical box } \\
\hline $\mathrm{BMD}\left(\mathrm{mg} / \mathrm{cm}^{3}\right)$ & $707 \pm 19$ & $698 \pm 16$ & $692 \pm 21$ & $723 \pm 15$ & $756 \pm 13$ * \\
\hline $\mathrm{BMC}(\mu \mathrm{g})$ & $14.9 \pm 0.7$ & $14.7 \pm 0.4$ & $14.9 \pm 0.8$ & $15.1 \pm 0.4$ & $15.6 \pm 0.4$ \\
\hline
\end{tabular}

FIGURE 5 | MDY treatment benefits cortical area and density. Top image is a representative isosurface of chick diaphyseal cortical bone demonstrating the $7 \mathrm{~mm}$ cube volume region and indicating where the small cortical cube $\left(0.7 \mathrm{~mm}^{3}\right)$ region is taken. Table represents measures as means \pm SE. $N \geq 15$ per group. ${ }^{*} p<0.05$ compared to Salmonella control; $\wedge p<0.01$ compared to Salmonella control as determined by 1 -way ANOVA with a Fischer ad hoc test.

in infection, can lead to the production of proinflammatory cytokines and alterations in the immune cell population that are important for reducing further infection but simultaneously can reduce anabolic processes such as bone formation (Harris et al., 2009). Interestingly, unlike previous reports which indicate that decreased bone formation is the main cause for bone loss in models of intestinal inflammation (Lin et al., 1996; Harris et al., 2009), Salmonella infection in this study lead to an increase in the number of multi-nucleated TRAP-positive osteoclasts on the bone surface. This finding points toward an increase in bone resorption as the cause for decreased bone density in Salmonellainfected chickens. Accordingly, inflammation, as would be seen with infection or colitis, is known to promote osteoclastogenesis.

Past studies have indicated a beneficial role for oral probiotics in reducing broiler chick lameness (Wideman and Prisby, 2012), though exact mechanisms are not known. Here we show that oral ingestion of MDY also benefits broiler bone health. In this study we chose two different treatment schedules, continuous (121 days) and acute (14-21 days), in order to determine if MDY was able to prevent bone loss or if it could reverse bone loss. We found that continous MDY treatment counteracts femoral neck bone loss, structural changes and the increase in osteoclast surface caused by Salmonella infection. Even more impressive is our finding that acute MDY treatment, beginning 7 days after
Salmonella infection, was able to prevent the decreased bone density in both the femoral neck as well as cortical bone. We did not measure bone density 1 week after Salmonella infection. If presumably adverse bone changes were occurring within the first week of infection, MDY treatment may be able to reverse bone loss. Future studies will investigate the latter postulate on MDY efficacy. Additionally, it will be important for future studies to examine longer-term effects of infection on the chicken skeleton. For example, if we extended the time course of the current study, infected chickens may continue to display increased bone loss (which could affect the entire femur bone trabecular structure); however, it is also possible that with time the bone loss we observe is regained following control of the infection.

While we do not know the exact mechanisms accounting for the beneficial effects of MDY on chicken bone health, our data indicate that MDY does not work by eradicating Salmonella. This is demonstrated by the high Salmonella recovery rates from the ceca of MDY treated birds. This suggests, that MDY may be preventing the negative gut-bone signaling in responses to Salmonella infection. While our findings indicate that altered regulation of osteoclast activity contributes to the mechanism of Salmonella-induced bone loss and to MDY prevention of bone loss, additional analyses are needed (such as intestinal cytokine profiles, histology and intestinal permeability) to identify the 
A

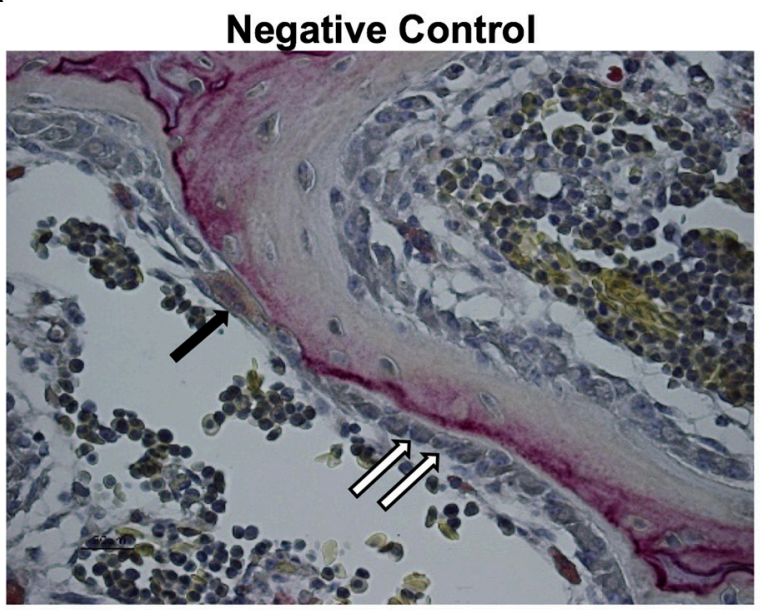

Salmonella Control

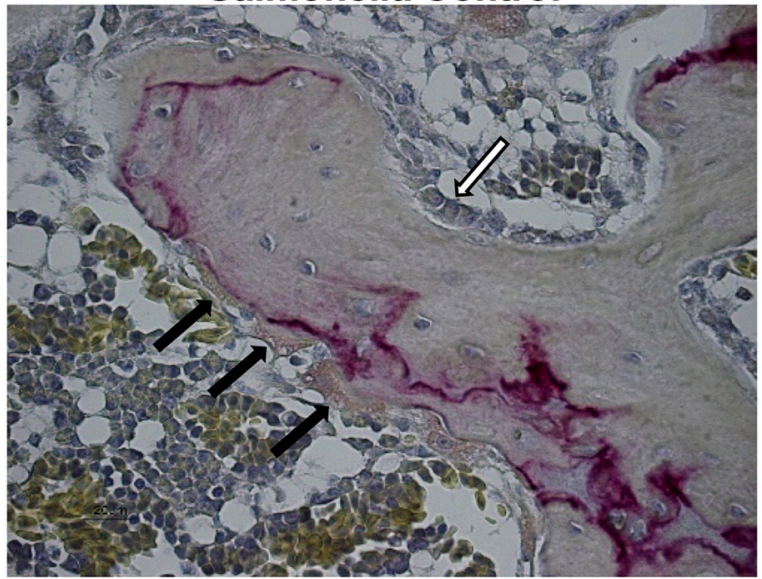

Salmonella + MDY (1-21d)

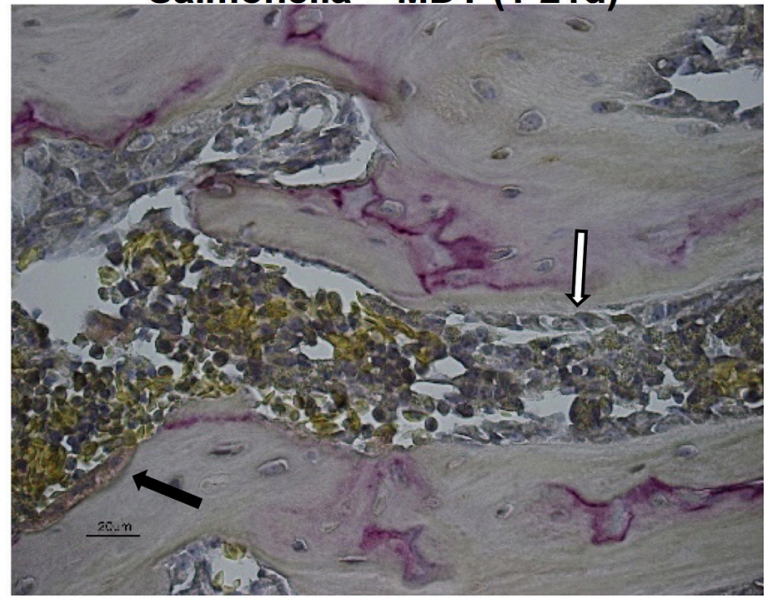

B

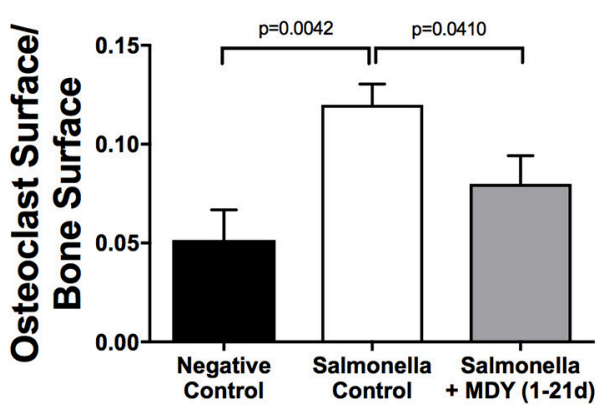

C

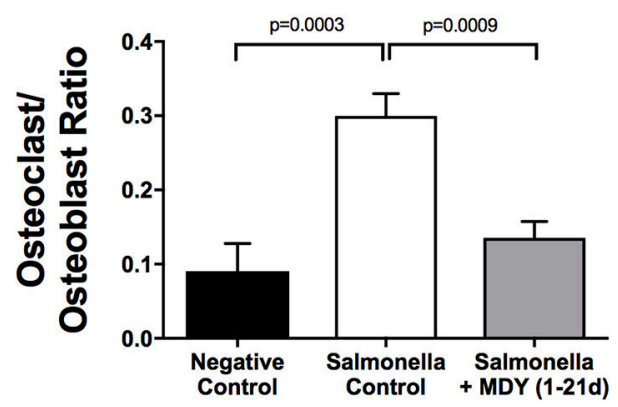

D

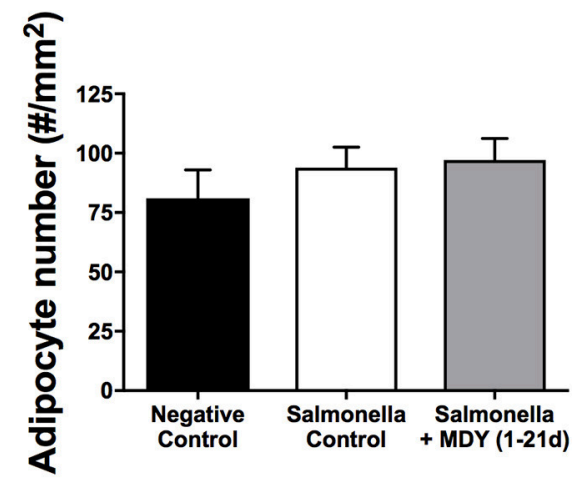

E

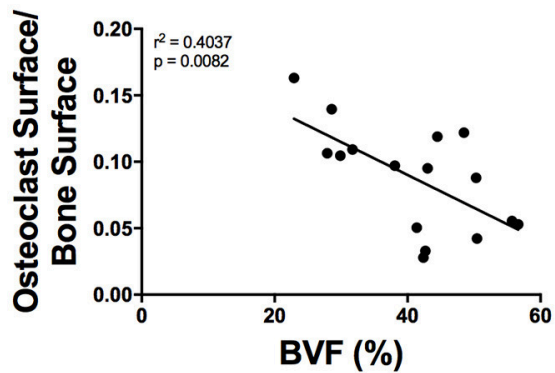

$\mathbf{F}$

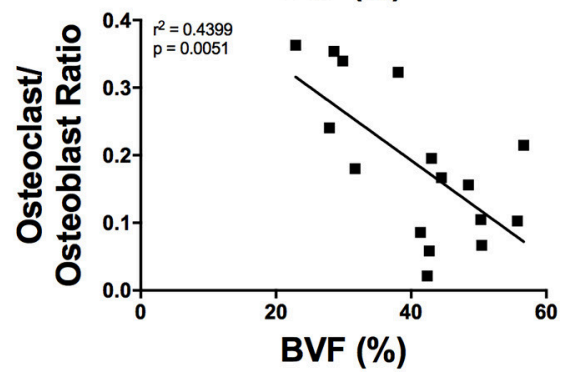

FIGURE 6 | MDY prevents Salmonella-induced increase in osteoclast surface and osteoclast to osteoblast ratio. (A) Representative TRAP-stained, 40X images of the femoral neck region. Black arrows indicate TRAP-positive osteoclasts; White arrows indicate cuboidal osteoblasts. (B) Ratio of osteoclast surface over total bone surface measured in chick femurs stained with TRAP. (C) Ratio of osteoclast to osteoblast number. (D) Quantification of bone marrow adipocytes. (E,F) Pearson Correlation analyses of osteoclast surface/bone surface or osteoclast/osteoblast number to bone volume fraction (BVF). Values are means \pm SE. $N=6$ per group. Lines represent statistical differences $(p<0.05)$ between bars at ends of line as determined by 1 -way ANOVA with a Fischer ad hoc test. 
specific mechanisms by which MDY influences the gut to cause the changes in bone. Gut inflammation, altered microbiota composition and increased barrier function are several key intestinal parameters that contribute to bone loss in mammals (Lin et al., 1996; Sylvester, 2005; Hamdani et al., 2008; Harris et al., 2009; Irwin et al., 2013, 2016; Mccabe et al., 2013; McCabe et al., 2015; Sylvester et al., 2013; Collins et al., 2017a,b; RiosArce et al., 2017; Schepper et al., 2017) and in poultry (Huff et al., 2006; Tellez et al., 2014; Wideman, 2016; Bielke et al., 2017). Past studies indicate that PEG derivatives can benefit intestinal health by increasing barrier function, reduce intestinal inflammation and contributing to overall host health (Wu et al., 2004; Valuckaite et al., 2013). Therefore, based on the above reports, it is likely that MDY influences the enteric health through multiple mechanisms that involve enhancing barrier function and reducing inflammation. Taken together, our work and the work of others indicate that orally ingested MDY prevents and possibly reverses the Salmonella-induced, negative effects on femoral bone density and architecture in broiler chickens and consequently be an effective way to promote chick bone health.

\section{REFERENCES}

Alexander, E. H., Bento, J. L., Hughes, F. M., Marriott, I., Hudson, M. C., and Bost, K. L. (2001). Staphylococcus aureus and Salmonella enterica serovar dublin induce tumor necrosis factor-related apoptosis-inducing ligand expression by normal mouse and human osteoblasts. Infect. Immun. 69, 1581-1586. doi: 10.1128/IAI.69.3.1581-1586.2001

Bielke, L. R., Hargis, B. M., and Latorre, J. D. (2017). Impact of enteric health and mucosal permeability on skeletal health and lameness in poultry. Adv. Exp. Med. Biol. 1033, 185-197. doi: 10.1007/978-3-319-6665 3-2_9

Butterworth, A., Reeves, N. A., Harbour, D., Werrett, G., and Kestin, S. C. (2001). Molecular typing of strains of staphylococcus aureus isolated from bone and joint lesions in lame broilers by random amplification of polymorphic DNA. Poult. Sci. 80, 1339-1343. doi: 10.1093/ps/80.9.1339

Carey, C. M., and Kostrzynska, M. (2013). Lactic acid bacteria and bifidobacteria attenuate the proinflammatory response in intestinal epithelial cells induced by Salmonella enterica serovar Typhimurium. Can. J. Microbiol. 59, 9-17. doi: 10.1139/cjm-2012-0446

Collins, F. L., Irwin, R., Bierhalter, H., Schepper, J., Britton, R. A., Parameswaran, N., et al. (2016). Lactobacillus reuteri 6475 increases bone density in intact females only under an inflammatory setting. PLoS ONE 11:e0153180. doi: 10.1371/journal.pone.0153180

Collins, F. L., Rios-Arce, N. D., Schepper, J. D., Parameswaran, N., and McCabe, L. R. (2017a). The potential of probiotics as a therapy for osteoporosis. Microbiol. Spectr. 5, 213-233. doi: 10.1128/microbiolspec.BAD-001 5-2016

Collins, F. L., Schepper, J. D., Rios-Arce, N. D., Steury, M. D., Kang, H. J., Mallin, H., et al. (2017b). Immunology of gut-bone signaling. Adv. Exp. Med. Biol. 1033, 59-94. doi: 10.1007/978-3-319-66653-2_5

Cook, M. E. (2000). Skeletal deformities and their causes: introduction. Poult. Sci. 79, 982-984. doi: 10.1093/ps/79.7.982

Corr, S. A., Maxwell, M., Gentle, M. J., and Bennett, D. (2003). Preliminary study of joint disease in poultry by the analysis of synovial fluid. Vet. Rec. 152, 549-554. doi: $10.1136 /$ vr.152.18.549

Dinev, I. (2009). Clinical and morphological investigations on the prevalence of lameness associated with femoral head necrosis in broilers. Br. Poult. Sci. 50, 284-290. doi: 10.1080/00071660902942783

Durairaj, V., Okimoto, R., Rasaputra, K., Clark, F. D., and Rath, N. C. (2009). Histopathology and serum clinical chemistry evaluation of

\section{AUTHOR CONTRIBUTIONS}

SR, BH, VK, LB, and LM have nothing to disclose. RP holds financial interest in Midway Pharmaceuticals, Inc. and Midway Animal Sciences. Midway Pharmaceuticals, Inc. and Midway Animal Sciences contributed MDY and methods of dosing and delivery but were not involved in the overall study design or collection, analysis or interpretation of the data.

\section{ACKNOWLEDGMENTS}

The authors thank the Biomedical Imaging Center at Michigan State University for use of the microcomputed tomography imaging equipment. The authors would also like to thank the Investigative Histology Laboratory in the Department of Physiology, Division of Human Pathology at Michigan State University for aiding us with histologic analyses. The authors thank Heather Mallin for her technical contributions to the manuscript. These studies were supported by funding from the National Institute of Health, grants RO1 DK101050 and RO1 AT007695 and by funding from Midway Animal Science.

broilers with femoral head separation disorder. Avian Dis. 53, 21-25. doi: 10.1637/8367-051908-Reg.1

Eckmann, L., and Kagnoff, M. F. (2001). Cytokines in host defense against Salmonella. Microbes Infect. 3, 1191-1200. doi: 10.1016/ S1286-4579(01)01479-4

Haghighi, H. R., Abdul-Careem, M. F., Dara, R. A., Chambers, J. R., and Sharif, S. (2008). Cytokine gene expression in chicken cecal tonsils following treatment with probiotics and Salmonella infection. Vet. Microbiol. 126, 225-233. doi: 10.1016/j.vetmic.2007.06.026

Hamdani, G., Gabet, Y., Rachmilewitz, D., Karmeli, F., Bab, I., and Dresner-Pollak, R. (2008). Dextran sodium sulfate-induced colitis causes rapid bone loss in mice. Bone 43, 945-950. doi: 10.1016/j.bone.2008. 06.018

Harris, L., Senagore, P., Young, V. B., and McCabe, L. R. (2009). Inflammatory bowel disease causes reversible suppression of osteoblast and chondrocyte function in mice. Am. J. Physiol. Gastrointest. Liver Physiol. 296, G1020-G1029. doi: 10.1152/ajpgi.90696.2008

Higgins, S. E., Wolfenden, A. D., Tellez, G., Hargis, B. M., and Porter, T. E. (2011). Transcriptional profiling of cecal gene expression in probiotic- and Salmonellachallenged neonatal chicks. Poult. Sci. 90, 901-913. doi: 10.3382/ps.201000907

Huff, G., Huff, W., Rath, N., Balog, J., Anthony, N. B., and Nestor, K. (2006). Stress-induced colibacillosis and turkey osteomyelitis complex in turkeys selected for increased body weight. Poult. Sci. 85, 266-272. doi: 10.1093/ps/85. 2.266

Irwin, R., Lee, T., Young, V. B., Parameswaran, N., and McCabe, L. R. (2013). Colitis-induced bone loss is gender dependent and associated with increased inflammation. Inflamm. Bowel Dis. 19, 1586-1597. doi: 10.1097/MIB.0b013e318289e17b

Irwin, R., Raehtz, S., Parameswaran, N., and McCabe, L. R. (2016). Intestinal inflammation without weight loss decreases bone density and growth. Am. J. Physiol. Regul. Integr. Comp. Physiol. 311, R1149-R1157. doi: 10.1152/ajpregu.00051.2016

Kaiser, P., Rothwell, L., Galyov, E. E., Barrow, P. A., Burnside, J., and Wigley, P. (2000). Differential cytokine expression in avian cells in response to invasion by Salmonella typhimurium, Salmonella enteritidis and Salmonella gallinarum. Microbiology 146 (Pt 12), 3217-3226. doi: 10.1099/00221287-14612-3217

Kebede, F. (2010). Pseudomonas infection in chickens. J. Vet. Med. Anim. Heal. 2, 55-58. doi: 10.5897/JVMAH 
Kestin, S. C., Gordon, S., Su, G., and Sorensen, P. (2001). Relationships in broiler chickens between lameness, liveweight, growth rate and age. Vet. Rec. 148, 195-197. doi: 10.1136/vr.148.7.195

Knowles, T. G., Kestin, S. C., Haslam, S. M., Brown, S. N., Green, L. E., Butterworth, A., et al. (2008). Leg disorders in broiler chickens: prevalence, risk factors and prevention. PLOS ONE 3:e1545. doi: 10.1371/journal.pone.00 01545

Kogut, M. H., McGruder, E. D., Hargis, B. M., Corrier, D. E., and Deloach, J. R. (1995). Characterization of the pattern of inflammatory cell influx in chicks following the intraperitoneal administration of live Salmonella enteritidis and Salmonella enteritidis-immune lymphokines. Poult. Sci. 74, 8-17. doi: 10.3382/ps.0740008

Kogut, M. H., Tellez, G. I., McGruder, E. D., Hargis, B. M., Williams, J. D., Corrier, D. E., et al. (1994). Heterophils are decisive components in the early responses of chickens to Salmonella enteritidis infections. Microb. Pathog. 16, 141-151. doi: 10.1006/mpat.1994.1015

Koob, A. O., and Borgens, R. B. (2006). Polyethylene glycol treatment after traumatic brain injury reduces $\beta$-amyloid precursor protein accumulation in degenerating axons. J. Neurosci. Res. 83, 1558-1563. doi: 10.1002/jnr. 20837

Kubota, T., Shimojo, N., Nonaka, K., Yamashita, M., Ohara, O., Igoshi, Y., et al. (2013). Prebiotic consumption in pregnant and lactating women increases IL-27 expression in human milk. Br. J. Nutr. 111, 625-632. doi: $10.1017 /$ S0007114513003036

Lin, C. L., Moniz, C., Chambers, T. J., and Chow, J. W. (1996). Colitis causes bone loss in rats through suppression of bone formation. Gastroenterology 111, 1263-1271. doi: 10.1053/gast.1996.v111.pm88 98640

Luo, J., and Shi, R. (2007). Polyethylene glycol inhibits apoptotic cell death following traumatic spinal cord injury. Brain Res. Rev. 1155, 10-16. doi: 10.1016/j.brainres.2007.03.091

Marriott, I. (2013). Apoptosis-associated uncoupling of bone formation and resorption in osteomyelitis. Front. Cell. Infect. Microbiol. 3:101. doi: 10.3389/fcimb.2013.00101

McCabe, L., Britton, R. A., and Parameswaran, N. (2015). Prebiotic and probiotic regulation of bone health: role of the intestine and its microbiome. Curr. Osteoporos. Rep. 13, 363-371. doi: 10.1007/s11914-0150292-x

Mccabe, L. R., Irwin, R., Schaefer, L., and Britton, R. A. (2013). Probiotic use decreases intestinal inflammation and increases bone density in healthy male but not female mice. J. Cell. Physiol. 228, 1793-1798. doi: 10.1002/jcp. 24340

McNamee, P. T., McCullagh, J. J., Rodgers, J. D., Thorp, B. H., Ball, H. J., Connor, T. J., et al. (1999). Development of an experimental model of bacterial chondronecrosis with osteomyelitis in broilers following exposure to Staphylococcus aureus by aerosol, and inoculation with chicken anaemia and infectious bursal disease viruses. Avian Pathol. 28, 26-35. doi: 10.1080/03079459995019

McNamee, P. T., and Smyth, J. A. (2000). Bacterial chondronecrosis with osteomyelitis ('femoral head necrosis') of broiler chickens: A review. Avian Pathol. 29, 253-270. doi: 10.1080/030794500501 18386

Mehaisen, G. M., Eshak, M. G., Elkaiaty, A. M., Atta, A. M., Mashaly, M. M., and Abass, A. O. (2017). Comprehensive growth performance, immune function, plasma biochemistry, gene expressions and cell death morphology responses to a daily corticosterone injection course in broiler chickens. PLoS ONE 12:e0172684. doi: 10.1371/journal.pone.0172684

Mireles, A. J., Kim, S. M., and Klasing, K. C. (2005). An acute inflammatory response alters bone homeostasis, body composition, and the humoral immune response of broiler chickens. Poult. Sci. 84, 553-560. doi: 10.1093/ps/84. 4.553

Moeser, A. J., Nighot, P. K., Roerig, B., Ueno, R., and Blikslager, A. T. (2008). Comparison of the chloride channel activator lubiprostone and the oral laxative Polyethylene Glycol 3350 on mucosal barrier repair in ischemic-injured porcine intestine. World J. Gastroenterol. 14, 6012-6017. doi: 10.3748/wjg. 14.6012

Neish, A. S. (2009). Microbes in gastrointestinal health and disease. Gastroenterology 136, 65-80. doi: 10.1053/j.gastro.2008.10.080
Olkowski, A. A., Laarveld, B., Wojnarowicz, C., Chirino-Trejo, M., Chapman, D., Wysokinski, T. W., et al. (2011). Biochemical and physiological weaknesses associated with the pathogenesis of femoral bone degeneration in broiler chickens. Avian Pathol. 40, 639-650. doi: 10.1080/03079457.2011. 626017

Oltean, M., Hellström, M., Ciuce, C., Zhu, C., and Casselbrant, A. (2015). Luminal solutions protect mucosal barrier during extended preservation. J. Surg. Res. 194, 289-296. doi: 10.1016/j.jss.2014.10.001

Oltean, M., Joshi, M., Björkman, E., Oltean, S., Casselbrant, A., Herlenius, G., et al. (2012). Intraluminal polyethylene glycol stabilizes tight junctions and improves intestinal preservation in the rat. Am. J. Transplant 12, 2044-2051. doi: 10.1111/j.1600-6143.2012.04067.x

Quinteiro-Filho, W. M., Gomes, A. V. S., Pinheiro, M. L., Ribeiro, A., Ferraz-de-Paula, V., Astolfi-Ferreira, C. S., et al. (2012). Heat stress impairs performance and induces intestinal inflammation in broiler chickens infected with Salmonella Enteritidis. Avian Pathol. 41, 421-427. doi: 10.1080/03079457.2012.709315

Quinteiro-Filho, W. M., Ribeiro, A., Ferraz-de-Paula, V., Pinheiro, M. L., Sakai, M., Sá, L. R., et al. (2010). Heat stress impairs performance parameters, induces intestinal injury, and decreases macrophage activity in broiler chickens. Poult. Sci. 89, 1905-1914. doi: 10.3382/ps.2010-00812

Rios-Arce, N. D., Collins, F. L., Schepper, J. D., Steury, M. D., Raehtz, S., Mallin, H., et al. (2017). Epithelial barrier function in gut-bone signaling. Adv. Exp. Med. Biol. 1033, 151-183. doi: 10.1007/978-3-319-6665 3-2_8

Schepper, J. D., Irwin, R., Kang, J., Dagenais, K., Lemon, T., Shinouskis, A., et al. (2017). Probiotics in gut-bone signaling. Adv. Exp. Med. Biol. 1033, 225-247. doi: 10.1007/978-3-319-66653-2_11

Setta, A. M., Barrow, P. A., Kaiser, P., and Jones, M. A. (2012). Early immune dynamics following infection with Salmonella enterica serovars enteritidis, infantis, pullorum and gallinarum: cytokine and chemokine gene expression profile and cellular changes of chicken cecal tonsils. Comp. Immunol. Microbiol. Infect. Dis. 35, 397-410. doi: 10.1016/j.cimid.2012. 03.004

Stabler, J. G., McCormick, T. W., Powell, K. C., and Kogut, M. H. (1994). Avian heterophils and monocytes: phagocytic and bactericidal activities against Salmonella enteritidis. Vet. Microbiol. 38, 293-305. doi: 10.1016/0378-1135(94)90148-1

Sylvester, F. A. (2005). IBD and skeletal health: children are not small adults! Inflamm. Bowel Dis. 11, 1020-1023. doi: 10.1097/01.MIB.0000188341.96 726.15

Sylvester, F. A., Gordon, C. M., Thayu, M., Burnham, J. M., Denson, L. A., Essers, J., et al. (2013). Report of the CCFA pediatric bone, growth and muscle health workshop, New York City, November 11-12, 2011, with updates. Inflamm. Bowel Dis. 19, 2919-2926. doi: 10.1097/MIB.0b013e3182a $5 \mathrm{a} 004$

Tellez, G., Latorre, J. D., Kuttappan, V. A., Kogut, M. H., Wolfenden, A., Hernandez-Velasco, X., et al. (2014). Utilization of rye as energy source affects bacterial translocation, intestinal viscosity, microbiota composition, and bone mineralization in broiler chickens. Front. Genet. 5:339. doi: 10.3389/fgene.2014.00339

Teramura, Y., Kaneda, Y., Totani, T., and Iwata, H. (2008). Behavior of synthetic polymers immobilized on a cell membrane. Biomaterials 29, 1345-1355. doi: 10.1016/j.biomaterials.2007.11.048

Valuckaite, V., Seal, J., Zaborina, O., Tretiakova, M., Testa, G., and Alverdy, J. C. (2013). High molecular weight polyethylene glycol (PEG 15-20) maintains mucosal microbial barrier function during intestinal graft preservation. J. Surg. Res. 183, 869-875. doi: 10.1016/j.jss.2013.02.035

Valuckaite, V., Zaborina, O., Long, J., Hauer-Jensen, M., Wang, J., Holbrook, C., et al. (2009). Oral PEG 15-20 protects the intestine against radiation: role of lipid rafts. AJP Gastrointest. Liver Physiol. 297, G1041-G1052. doi: 10.1152/ajpgi.00328.2009

Vestergaard, K. S., and Sanotra, G. S. (1999). Relationships between leg disorders and changes in the behaviour of broiler chickens. Vet. Rec. 144, 205-209. doi: 10.1136/vr.144.8.205

Wideman, R. F. (2016). Bacterial chondronecrosis with osteomyelitis and lameness in broilers: a review. Poult. Sci. 95, 325-344. doi: 10.3382/ps/ pev320 
Wideman, R. F., and Prisby, R. D. (2012). Bone circulatory disturbances in the development of spontaneous bacterial chondronecrosis with osteomyelitis: a translational model for the pathogenesis of femoral head necrosis. Front. Endocrinol. 3:183. doi: 10.3389/fendo.2012.00183

Withanage, G. S., Wigley, P., Kaiser, P., Mastroeni, P., Brooks, H., Powers, C., et al. (2005). Cytokine and chemokine responses associated with clearance of a primary Salmonella enterica serovar typhimurium infection in the chicken and in protective immunity to rechallenge. Infect. Immun. 73, 5173-5182. doi: 10.1128/IAI.73.8.5173-518 2.2005

Wu, L., Zaborina, O., Zaborin, A., Chang, E. B., Musch, M., Holbrook, C., et al. (2004). High-molecular-weight polyethylene glycol prevents lethal sepsis due to intestinal Pseudomonas aeruginosa. Gastroenterology 126, 488-498. doi: 10.1053/j.gastro.2003.11.011
Conflict of Interest Statement: RP holds financial interest in Midway Pharmaceuticals, Inc. and Midway Animal Sciences.

The other authors declare that the research was conducted in the absence of any commercial or financial relationships that could be construed as a potential conflict of interest.

Copyright (c) 2018 Raehtz, Hargis, Kuttappan, Pamukcu, Bielke and McCabe. This is an open-access article distributed under the terms of the Creative Commons Attribution License (CC BY). The use, distribution or reproduction in other forums is permitted, provided the original author(s) and the copyright owner are credited and that the original publication in this journal is cited, in accordance with accepted academic practice. No use, distribution or reproduction is permitted which does not comply with these terms. 